\title{
REGULAR REPRESENTATIONS OF DIRICHLET SPACES
}

\author{
BY \\ MASATOSHI FUKUSHIMA
}

\begin{abstract}
We construct a regular and a strongly regular Dirichlet space which are equivalent to a given Dirichlet space in the sense that their associated function algebras are isomorphic and isometric. There is an appropriate strong Markov process called a Ray process on the underlying space of each strongly regular Dirichlet space.
\end{abstract}

1. Introduction. A. Beurling and J. Deny [1] introduced the notion of Dirichlet spaces and developed the general theory of kernel-free potentials. Recently the author [6] adopted Dirichlet spaces relative to $L^{2}$-spaces (we will call them $L^{2}$ Dirichlet spaces or $D$-spaces as an abbreviation) to describe boundary conditions for multidimensional Brownian motions.

A $D$-space is a certain space of functions that are defined on an underlying measure space $(X, m)$. When $(X, m)$ is fixed, there is a one-to-one correspondence between the set of all symmetric sub-Markov resolvent operators on $L^{2}(X ; m)$ and the set of all $D$-spaces. In particular, any sub-Markov resolvent kernel on $X$ which is symmetric with respect to $m$ generates a $D$-space. The present paper and the subsequent one [9] concern the problem of whether conversely any $D$-space guarantees the existence of a suitable strong Markov process or not.

The present paper aims at constructing a regular and a strongly regular $D$-space which are equivalent to a given $D$-space. A $D$-space is called regular if it densely contains sufficiently many continuous functions vanishing at infinity on its underlying space. There corresponds a potential theory of a type of Beurling-Deny to each regular $D$-space. A strongly regular $D$-space is a regular one which is generated by a Ray resolvent kernel. According to D. Ray [15], there is a right continuous strong Markov process on the underlying space of each strongly regular $D$-space.

Suppose that we are given a $D$-space with underlying space $(X, m)$. Theorem 2 in $\S 5$ states that there exists then a regular $D$-space with some modified underlying space $\left(X^{\prime}, m^{\prime}\right)$ in such a way that these two $D$-spaces are equivalent to each other as function spaces. The latter $D$-space will be called a regular representation of the given one. The regular representation will be carried out depending on a subalgebra $L$ of $L^{\infty}(X ; m)$ satisfying a certain condition denoted by (C). Actually we will take as $X^{\prime}$ the space of all regular maximal ideals of $L$.

Received by the editors February 15, 1970.

AMS 1969 subject classifications. Primary 6060; Secondary 4655.

Key words and phrases. $D$-space, underlying space, $L^{2}$-resolvent, regular $D$-space, Ray resolvent, strongly regular $D$-space, Ray process, regular representation, continuous embedding. 
There are generally many possibilities to find $L$ satisfying (C). In $\S 6$, a special $L$ possessing an additional property denoted by $(R)$ will be constructed by making use of the method of F. Knight [11] and H. Kunita and T. Watanabe [12]. We can regard the condition ( $R$ ) as a generalization of Ray's hypothesis for a sub-Markov resolvent [15]. Theorem 3 in $\$ 6$ asserts that the regular representation with respect to such an $L$ turns out to be a strongly regular $D$-space.

$\S 3$ consists of typical examples of $D$-spaces related to the multidimensional Brownian motion. Those $D$-spaces except for the last example took the fundamental roles in the investigations of boundary problems by J. L. Doob [4] and by the author [5], [6]. The last example is a rather sophisticated one of regular $D$-spaces $\left({ }^{1}\right)$. Much stress on the roles of regular ones will be laid in [9].

The appendix is referred to only in $\$ 3$.

\section{Basic properties of $D$-spaces.}

Definition 2.1. We call $(X, m, \mathscr{F}, \mathscr{E})$ an $L^{2}$-Dirichlet space (or a $D$-space, for short) if the following conditions are satisfied.

(D.1) $X$ is a locally compact, Hausdorff, and separable space. $m$ is a Radon measure on $X$.

(D.2) $\mathscr{F}$ is a linear subspace of the real $L^{2}(X)=L^{2}(X ; m)$, two functions of $\mathscr{F}$ being identified if they coincide $m$-a.e. on $X$. $\mathscr{E}$ is a symmetric nonnegative definite bilinear form on $\mathscr{F}$ and, for each $\alpha>0, \mathscr{F}$ is a real Hilbert space with respect to the inner product

$$
\mathscr{E}^{\alpha}(u, v)=\mathscr{E}(u, v)+\alpha(u, v)_{X}, \quad u, v \in \mathscr{F}
$$

where $(u, v)_{X}$ denotes the inner product of $L^{2}(X)$.

(D.3) Every normal contraction operates on $(\mathscr{F}, \mathscr{E}):$ if $u \in \mathscr{F}$ and a $m$-measurable function $v$ satisfies inequalities

$$
|v(x)| \leqq|u(x)|, \quad|v(x)-v(y)| \leqq|u(x)-u(y)|
$$

$m$-a.e. on $X$, then $v \in \mathscr{F}$ and $\mathscr{E}(v, v) \leqq \mathscr{E}(u, u)$.

The present definition of $D$-space was given in [6]. $(X, m)$ is called the underlying space of the $D$-space. According to $\$ 2$ of [6], let us state a theorem about a one-to-one correspondence between $D$-spaces and $L^{2}$-resolvents.

Definition 2.2. Let $(X, m)$ satisfy condition (D.1). A system $\left\{G_{\alpha}, \alpha>0\right\}$ of linear, bounded and symmetric operators on $L^{2}(X)$ is called an $L^{2}$-resolvent if it has the following properties.

(G.1) Sub-Markov property: if $u \in L^{2}(X)$ and $0 \leqq u \leqq 1 m$-a.e. then $0 \leqq \alpha G_{\alpha} u \leqq 1$ $m$-a.e., for any $\alpha>0$.

(G.2) Resolvent equation: $G_{\alpha}-G_{\beta}+(\alpha-\beta) G_{\alpha} G_{\beta}=0, \alpha, \beta>0$.

( $\left.{ }^{1}\right) \mathrm{N}$. Ikeda suggested to the author the last example of $\$ 3$ and theorem of the appendix. 
THEOREM 1. Let us fix $(X, m)$ satisfying condition (D.1). For a given $D$-space $(\mathscr{F}, \mathscr{E})$ with underlying space $(X, m)$, there exists a unique $L^{2}$-resolvent $\left\{G_{\alpha}, \alpha>0\right\}$ on $L^{2}(X)$ satisfying the equation

$$
\mathscr{E}^{\alpha}\left(G_{\alpha} u, v\right)=(u, v)_{X}
$$

for any $v \in \mathscr{F}$, where $\alpha>0$ and $u \in L^{2}(X)$ are arbitrarily fixed. Conversely, for $a$ given $L^{2}$-resolvent $\left\{G_{\alpha}, \alpha>0\right\}$ on $L^{2}(X)$, a $D$-space is defined by

$$
\begin{aligned}
\mathscr{F} & =\left\{u \in L^{2}(X) ; \lim _{\beta \rightarrow+\infty} \beta\left(u-\beta G_{\beta} u, u\right)_{X}<+\infty\right\}, \\
\mathscr{E}(u, v) & =\lim _{\beta \rightarrow+\infty} \beta\left(u-\beta G_{\beta} u, v\right)_{X}, \quad u, v \in \mathscr{F} .
\end{aligned}
$$

The correspondence defined by (2.2) and that defined by (2.3) and (2.4) are reciprocal to each other.

REMARK 2.1. (i) The proof of Theorem 1 was sketched in $\$ 2$ of [6]. The essential ideas for the proof can be found in Beurling-Deny [1] and Deny [2]. So far as this theorem and the next lemma are concerned, condition (D.1) for $(X, m)$ can be much weakened. These have been proved in [7] without the separability assumption for $X$ (see also [8]). T. Shiga and T. Watanabe [16] gave a detailed proof of Theorem 1 under the assumption that, instead of (D.1), the underlying space $(X, m)$ is merely a $\sigma$-finite measure space.

(ii) Condition (D.3) in the definition of $D$-space can be replaced with the following apparently weaker but equivalent condition (D.3)' [16].

(D.3)' Every unit contraction operates on $(\mathscr{F}, \mathscr{E}):$ if $u \in \mathscr{F}$ then $v=(0 \vee u) \wedge 1$ is also in $\mathscr{F}$ and $\mathscr{E}(v, v) \leqq \mathscr{E}(u, u)$. Here, the lattice operations $\vee$ and $\wedge$ for functions on $X$ are defined by $\left(u_{1} \vee u_{2}\right)(x)=\max \left(u_{1}(x), u_{2}(x)\right)$ and $u_{1} \wedge u_{2}=-\left(\left(-u_{1}\right) \vee\left(-u_{2}\right)\right)$.

The next lemma states the basic properties of $D$-spaces which we need in the later discussions. Notice that, for a $D$-space, $\mathscr{E}^{\alpha}$ and $\mathscr{E}^{\beta}$ define equivalent metrics on $\mathscr{F}$ for any $\alpha, \beta>0$.

Lemma 2.1. Let $(X, m, \mathscr{F}, \mathscr{E})$ be a $D$-space and $\left\{G_{\alpha}, \alpha>0\right\}$ be its associated $L^{2}$ resolvent. Fix an $\alpha_{0}>0$.

(i) If $S$ is a dense subset of $L^{2}(X)$, then, for any $\alpha>0, G_{\alpha}(S)$ is dense in $\mathscr{F}$ with respect to metric $\mathscr{E}^{\alpha}$.

(ii) For $u, v \in \mathscr{F}$,

$$
\mathscr{E}^{\alpha}(u, v)=\lim _{\beta \rightarrow+\infty} \beta\left(u-\beta G_{\beta+\alpha} u, v\right)_{X}
$$

(iii) For any $u \in \mathscr{F}, \lim _{\beta \rightarrow+\infty} \beta G_{\beta} u=u$ strongly in norm $\mathscr{E}^{\alpha_{0}}$ and hence strongly in $L^{2}(X)$ sense.

(iv) $\mathscr{F}$ is a function lattice: if $u, v \in \mathscr{F}$, then $u \vee v, u \wedge v \in \mathscr{F}$. Further $u \wedge 1 \in \mathscr{F}$ for $u \in \mathscr{F}$. 
(v) If $u$ and $v$ are both in $\mathscr{F}$ and m-essentially bounded, then the product $u \cdot v$ is also in $\mathscr{F}$.

(vi) For $u \in \mathscr{F}$, put $u_{n}=((-n) \vee u) \wedge n$.

Then $\lim _{n \rightarrow+\infty} u_{n}=u$ strongly in norm $\mathscr{E} \alpha_{0}$.

Proof. (i) is a consequence of the equation (2.2).

(ii) is a consequence of Lemma 1 of [8].

(iii) For $\beta>\alpha_{0}$,

$$
\begin{aligned}
\mathscr{E}_{0} \alpha_{0}\left(\beta G_{\beta} u-u, \beta G_{\beta} u-u\right) & \leqq \mathscr{E}^{\beta}\left(\beta G_{\beta} u-u, \beta G_{\beta} u-u\right) \\
& =\beta^{2}\left(\beta G_{\beta} u, u\right)_{X}-2 \beta(u, u)_{X}+\mathscr{E}^{\beta}(u, u) \\
& =-\beta\left(u-\beta G_{\beta} u, u\right)_{X}+\mathscr{E}(u, u) \rightarrow 0, \quad \beta \rightarrow+\infty .
\end{aligned}
$$

(iv) Since $|u|$ and $u \wedge 1$ are normal contractions of $u$, they are in $\mathscr{F}$ if $u$ is. Note that

$$
u \vee v=\frac{1}{2}((u+v)+|u-v|), \quad u \wedge v=\frac{1}{2}((u+v)-|u-v|) .
$$

(v) If $u \in \mathscr{F}$ and $|u| \leqq M m$-a.e. for some constant $M$, then $u^{2}$ is a normal contraction of $2 M u$ and hence $u^{2} \in \mathscr{F}$. Note that $u \cdot v=\frac{1}{4}\left((u+v)^{2}-(u-v)^{2}\right)$.

(vi) By Lemma 2.1 of [6], $\mathscr{E}^{\alpha} \alpha_{0}\left(u_{n}, u_{n}\right)$ increases to $\mathscr{E}^{\alpha} 0(u, u)$ as $n$ tends to infinity. On the other hand,

$$
\mathscr{E}^{\alpha_{0}}\left(u_{n}, G_{\alpha_{0}} w\right)=\left(u_{n}, w\right)_{X} \underset{n \rightarrow+\infty}{\longrightarrow}(u, w)_{X}=\mathscr{E}^{\alpha_{0}}\left(u, G_{\alpha_{0}} w\right)
$$

for any $w \in L^{2}(X)$. These facts combined with the first statement of this lemma imply that $u_{n}$ converges to $u$ weakly and after all strongly with respect to the inner product $\mathscr{E}^{\alpha_{0}}$.

We will now give definitions and remarks concerning regularity of $D$-spaces. For a locally compact space $X$, denote by $C(X)$ (resp. $C_{0}(X)$ ) the space of all continuous functions vanishing at infinity (resp. with compact supports). $C^{+}(X)$ (resp. $C_{0}^{+}(X)$ ) will denote the set of all nonnegative elements of $C(X)$ (resp. $C_{0}(X)$ ). We say a measure $m$ on $X$ to be everywhere dense if $m(E)$ is not zero for any nonempty open set $E \subset X$.

Definition 2.3. A $D$-space $(X, m, \mathscr{F}, \mathscr{E})$ is called regular if $m$ is everywhere dense and $\mathscr{F} \cap C(X)$ is dense both in $\mathscr{F}$ with norm $\mathscr{E}^{\alpha_{0}}$ and in $C(X)$ with uniform norm. Here, $\alpha_{0}>0$ is arbitrarily fixed.

Next, consider $(X, m)$ satisfying condition (D.1). For a sub-Markov resolvent $\operatorname{kernel}\left({ }^{2}\right)\left\{G_{\alpha}(x, E), \alpha>0\right\}$ on $X$, we set

$$
G_{\alpha} u(x)=\int_{x} G_{\alpha}(x, d y) u(y), \quad u \in C(X) .
$$

$\left({ }^{2}\right) G_{\alpha}(x, E)$ is called a kernel on $X$ if, for a fixed $x \in E, G_{\alpha}(x, \cdot)$ is a Borel measure on $X$ and, for a fixed Borel set $E \subset X, G_{\alpha}(\cdot, E)$ is a measurable function on $X$. 
Definition 2.4. (i) A sub-Markov resolvent kernel $\left\{G_{\alpha}(x, E), \alpha>0\right\}$ on $X$ is called $m$-symmetric if

$$
\int_{x} G_{\alpha} u(x) \cdot v(x) m(d x)=\int_{x} u(x) \cdot G_{\alpha} v(x) m(d x) \leqq+\infty
$$

for any $u, v \in C^{+}(X)$. (ii) A sub-Markov resolvent kernel $\left\{G_{\alpha}(x, E), \alpha>0\right\}$ on $X$ is called a Ray resolvent if it satisfies the following conditions.

(R.a) $G_{\alpha}(C(X)) \subset C(X)$ for any $\alpha>0$.

(R.b) There exists a countable subcollection $C_{1}$ of $C^{+}(X)$ such that $(\alpha) C_{1}$ separates points of $X$, and, for any $x \in X$, there exists a $u \in C_{1}$ whose value at $x$ is not zero, $(\beta)$ for some $\alpha_{0}>0$, every function $u \in C_{1}$ satisfies the inequality $\beta G_{\alpha_{0}+\beta} u \leqq u, \beta>0$.

Consider any $m$-symmetric sub-Markov resolvent kernel $\left\{G_{\alpha}(x, E), \alpha>0\right\}$ on $X$. It satisfies the inequality $\left(\alpha G_{\alpha} u, \alpha G_{\alpha} u\right)_{X} \leqq(u, u)_{X}$ for all $u \in L^{2}(X ; m) \cap C(X)$ [16]. Therefore it determines a unique $L^{2}$-resolvent. The Dirichlet space associated with this $L^{2}$-resolvent will be said to be generated by the resolvent kernel $\left\{G_{\alpha}(x, E), \alpha>0\right\}$.

We will say the set $C_{1}$ appearing in the definition of Ray resolvent to be attached to the given Ray resolvent.

Definition 2.5. A $D$-space $(X, m, \mathscr{F}, \mathscr{E})$ is called strongly regular if $m$ is everywhere dense on $X,(\mathscr{F}, \mathscr{E})$ is generated by an $m$-symmetric Ray resolvent on $X$ and $\mathscr{F} \cap C(X)$ contains the set $C_{1}$ attached to this Ray resolvent.

REMARK 2.2. (i) A strongly regular $D$-space is regular. To see this, let $(X, m, \mathscr{F}, \mathscr{E})$ be a strongly regular $D$-space and $\left\{G_{\alpha}(x, E), \alpha>0\right\}$ be its associated Ray resolvent. $\mathscr{F} \cap C(X)$ contains $G_{\alpha}\left(L^{2}(X) \cap C(X)\right)$, which is dense in $\left(\mathscr{F}, \mathscr{E}^{\alpha_{0}}\right)$ by virtue of Lemma 2.1(i). Owing to the fifth statement of the lemma, $\mathscr{F} \cap C(X)$ is a function algebra. Since it contains the set $C_{1}$ attached to $\left\{G_{\alpha}, \alpha>0\right\}$, it is dense in $C(X)$ by Stone-Weierstrass theorem.

(ii) Consider a Ray resolvent $\left\{G_{\alpha}(x, E), \alpha>0\right\}$ on a locally compact Hausdorff separable space $X$. Let $\bar{X}=X \cup\{\infty\}$ be the one point compactification of $X$ if $X$ is not compact. If $X$ is compact, let $\{\infty\}$ be an isolated point. Define a new kernel $\left\{\bar{G}_{\alpha}(x, E), \alpha>0\right\}$ on $\bar{X}$ by $\bar{G}_{\alpha}(x, E)=G_{\alpha}(x, E \cap X)+\left(\left(1-\alpha G_{\alpha}(x, X)\right) / \alpha\right) \delta_{\{\infty\}}(E)$, $x \in X, \bar{G}_{\alpha}(\{\infty\}, E)=(1 / \alpha) \delta_{\{\infty\}}(E)$. Then $\left\{\bar{G}_{\alpha}, \alpha>0\right\}$ is a conservative Ray resolvent on the compactum $\bar{X}$. By Ray's theory [15], [12], this defines on $\bar{X}$ a right continuous conservative strong Markov process for which the point $\{\infty\}$ is a trap. Thus, we obtain a right continuous strong Markov process $\left(X_{t}, \zeta, P_{x}, x \in X\right)$ on $X$ such that

$$
G_{\alpha}(x, E)=E_{x}\left(\int_{0}^{\zeta} e^{-\alpha t} \chi_{E}\left(X_{t}\right) d t\right)
$$

$\chi_{E}$ being the indicator function of the Borel set $E$. We will call the process on $X$ so obtained the Ray process associated with the Ray resolvent $\left\{G_{\alpha}, \alpha>0\right\}$ on $X$.

There is a Ray process on the underlying space of any strongly regular $D$-space. 
3. Examples. Denote by $D$ a domain of Euclidean $N$-space $R^{N}(N \geqq 1)$.

EXAMPLE 1. Let us put

$$
\begin{aligned}
\mathscr{E}_{L^{2}}^{1}(D) & =\left\{u ; u \in L^{2}(D), \partial u / \partial x_{i} \in L^{2}(D), i=1,2, \ldots, N\right\}, \\
(u, v)_{D, 1} & =\frac{1}{2} \int_{D} \sum_{i=1}^{N} \frac{\partial u}{\partial x_{i}} \frac{\partial v}{\partial x_{i}} d x .
\end{aligned}
$$

Here, derivatives are taken in Schwartz distribution sense and $d x$ denotes the Lebesgue measure on $R^{N}$.

$\left(D, d x, \mathscr{E}_{L^{2}}^{12}(D),(,)_{D, 1}\right)$ is a $D$-space in our sense. Condition (D.3)' for this space can be verified easily (see Proposition A.1 of [16] or Théorème 3.1 of [3]). This space is not regular except when it coincides with $\mathscr{D}_{L^{2}}^{1}(D)$ of the next example. Denote by $\bar{D}$ the closure of $D$ in $R^{N}$. Let $C^{\infty}(\bar{D})$ be the space of restrictions to $\bar{D}$ of functions which are infinitely differentiable on $R^{N}$. If $\partial D=\bar{D}-D$ is a closed hypersurface of class $C^{1}$, then $\mathscr{E}_{L^{2}}^{1}(D) \cap C^{\infty}(\bar{D})$ is dense in $\mathscr{E}_{L^{2}}{ }^{2}(D)$ [14]. Therefore, in this case, $\left(\bar{D}, d x, \mathscr{E}_{L^{2}}^{1}(D),(,)_{D, 1}\right)\left({ }^{3}\right)$ is a regular $D$-space. When $D$ is bounded, the space $\left(\mathscr{E}_{L^{2}}^{1}(D),(,)_{D, 1}\right)$ is generated by the continuous resolvent density constructed in [5] and in $\S 8(\mathrm{I})$ of [6].

EXAMPLE 2. Denote by $C_{0}^{\infty}(D)$ the space of infinitely differentiable functions on $D$ with compact supports. Let $\mathscr{D}_{L}^{1}(D)$ be the closure of $C_{0}^{\infty}(D)$ in

$$
\left(\mathscr{E}_{L^{2}}^{1}(D),(,)_{D, 1}+(,)_{D}\right) \text {. }
$$

$\left(D, d x, \mathscr{D}_{L^{2}}^{1}(D),(,)_{D, 1}\right)$ is a regular $D$-space. Since $\mathscr{D}_{L^{2}}^{1}(D)$ coincides with the completion of $\mathscr{E}_{L^{2}}^{1}(D) \cap C_{0}(D)$ with respect to metric $(,)_{D ; 1}+(\text {, })_{D}$, we can apply Corollary 3 of Appendix to show that it is a regular $D$-space. It is generated by a continuous resolvent density of the absorbing barrier Brownian motion on $D$ [6]. It is strongly regular when each point of the boundary $\partial D$ is regular with respect to the Dirichlet problem for $D$.

Example 3. Let $M$ be the Martin boundary of the domain $D$ and $\mu$ be the harmonic measure on $M$ with respect to a reference point $x_{0}$ of $D$. J. L. Doob [4] introduced the space $\boldsymbol{H}_{h}^{\prime}$ of measurable functions $\varphi$ on $M$ for which the integral

$$
\boldsymbol{D}_{M}(\varphi, \varphi)=\frac{q}{4} \int_{M} \int_{M}(\varphi(\xi)-\varphi(\eta))^{2} \theta(\xi, \eta) \mu(d \xi) \mu(d \eta)
$$

is finite. Here, $\theta(\xi, \eta)$ is Naim's kernel on $M$ and $q$ is $2 \pi$ if $N=2$ or the product of $N-2$ and the unit ball boundary area if $N>2$. It was proved in [4] that $\boldsymbol{H}_{h}^{\prime} \subset$ $L^{2}(M ; \mu)$. We can easily see that $\left(M, \mu, \boldsymbol{H}_{h}^{\prime}, \boldsymbol{D}_{M}(),\right)$ is a $D$-space. This is regular when $D$ is a disk ( $\$ 18$ of [4]). Let $\boldsymbol{H}_{h}$ be the space of all harmonic functions on $D$ with finite integrals $(u, u)_{D, 1}$. Then, $\left(\boldsymbol{H}_{h}^{\prime}, \boldsymbol{D}_{M}(),\right)$ is the trace on $M$ of the space $\left(\boldsymbol{H}_{h},(,)_{D, 1}\right)$ in the following sense: each function $u$ of $\boldsymbol{H}_{h}$ has a fine boundary

$\left(^{3}\right)$ We regard here $\mathscr{E}_{L^{2}}^{1}(D)$ as a subspace of $L^{2}(\bar{D})\left(=L^{2}(D)\right)$. See Remark 5.2. 
limit function $\gamma u$ in $\boldsymbol{H}_{h}^{\prime}$ and $\gamma$ define a unitary map from $\left(\boldsymbol{H}_{h},(,)_{D, 1}\right)$ onto $\left(\boldsymbol{H}_{h}^{\prime}, \boldsymbol{D}_{M}\right)$. This is the reason why functions of $H_{h}^{\prime}$ were called in [4] BLD boundary functions.

A modification of the space $\left(\boldsymbol{H}_{h}^{\prime}, \boldsymbol{D}_{M}\right)$ was introduced in [6] in order to describe the space of all $\alpha$-harmonic functions of $\mathscr{E}_{L}^{1}{ }^{2}(D)$. Suppose that $D$ is bounded. Let $U_{\alpha}(\xi, \eta)$ and $U(\xi, \eta)$ be Feller kernels on $M . U(\xi, \eta)$ is equal to $(q / 2) \cdot \theta(\xi, \eta) \mu$-a.e. Denote by $\mu^{\prime}$ the measure $U_{1} 1 \cdot \mu$ on $M$ and put $\boldsymbol{H}_{M}=\boldsymbol{H}_{h}^{\prime} \cap L^{2}\left(M ; \mu^{\prime}\right)$. Then, $\left(M \mu^{\prime} H_{M}, D_{M}\right)$ is a $D$-space. By virtue of Lemma 3.1 and equality (3.21) of [6], it is clear that $\left(M, \mu^{\prime}, \boldsymbol{H}_{M}, \boldsymbol{D}_{M}^{[\alpha]}\right)$ is also a $D$-space for each $\alpha>0$, where

$$
\boldsymbol{D}_{M}^{[\alpha]}(\varphi, \psi)=\boldsymbol{D}_{M}(\varphi, \psi)+\int_{M} \int_{M} \varphi(\xi) U_{\alpha}(\xi, \eta) \psi(\eta) \mu(d \xi) \mu(d \eta)
$$

Let $\mathscr{H}_{\alpha}$ be the orthogonal complement of $\mathscr{D}_{L}^{1} 2(D)$ in the Hilbert space

$$
\left(\mathscr{E}_{L}^{1}{ }_{L}(D),(,)_{D, 1}+\alpha(,)_{D}\right) .
$$

The space $\left(\boldsymbol{H}_{M}, \boldsymbol{D}_{M}^{[\alpha]}\right)$ is nothing but the trace on $M$ of the space

$$
\left.\left(\mathscr{H}_{\alpha},(,)_{D, 1}+\alpha(,)_{D}\right){ }^{4}\right) \text {. }
$$

EXAmple $4\left({ }^{5}\right)$. Assume that $D$ is bounded. Let $\Delta=\bigcup_{p \in P} E_{p}$ be a measurable partition of the Martin boundary $M$. Then, $\Delta$ defines a Dirichlet subspace $\left(\mathscr{F}_{M}^{\Delta}, \boldsymbol{D}_{M}\right)$ of $\left(\boldsymbol{H}_{M}, \boldsymbol{D}_{M}\right)$ by $\mathscr{F}_{M}^{\Delta}=\left\{\varphi \in \boldsymbol{H}_{M}\right.$; there exists a set $E_{\varphi}$ such that $\mu^{\prime}\left(E_{\varphi}\right)=0$ and $\varphi$ is a constant on $E_{p}-E_{\varphi}$ for each $\left.p \in \boldsymbol{P}\right\}$. Even when $D$ is a unit disk, $\left(M, \mu^{\prime}, \mathscr{F}_{M}^{\Delta}, \boldsymbol{D}_{M}\right)$ is no longer regular except for a trivial case that $\mathscr{F}_{M}^{\Delta}$ is equal to $\boldsymbol{H}_{M}$.

EXAmple 5. Consider the whole plane $R^{2}$ and put

$$
\begin{aligned}
& \mathscr{E}(u, v)=\frac{1}{2} \int_{-\infty}^{+\infty} \int_{-\infty}^{+\infty}\left(\frac{\partial u}{\partial x} \frac{\partial v}{\partial x}+\frac{\partial u}{\partial y} \frac{\partial v}{\partial y}\right) d x d y+\frac{1}{2} \int_{-\infty}^{\infty} \frac{\partial u(x, 0)}{\partial x} \frac{\partial v(x, 0)}{\partial x} d x \\
& \mathscr{A}=\left\{u \in C_{0}\left(R^{2}\right) ; u(x, y) \text { is absolutely continuous in each variable } x \text { and } y\right. \\
&\text { and } \mathscr{E}(u, u)<+\infty\} .
\end{aligned}
$$

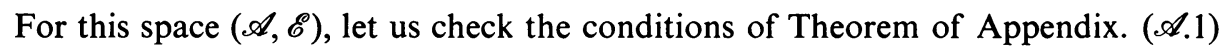

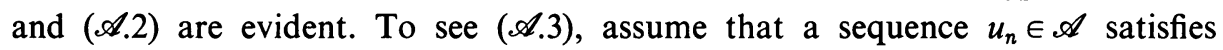
$\left(u_{n}, u_{n}\right)_{R^{2}} \rightarrow 0$ and $\mathscr{E}\left(u_{n}-u_{m}, u_{n}-u_{m}\right) \rightarrow 0$. We have to prove $\mathscr{E}\left(u_{n}, u_{n}\right) \rightarrow 0$. Since $u_{n}$ converges to zero in $\mathscr{D}_{L^{2}}^{1}\left(R^{2}\right)$ with metric $(,)_{R^{2}, 1}+(,)_{R}{ }^{2}$, we can select a subsequence $u_{n_{k}}$ such that $u_{n_{k}}(x, y)$ converges to zero for every $(x, y)$ except on a 2-dimensional Brownian polar set( $\left.{ }^{6}\right)$ [3]. Especially, $u_{n_{k}}(x, 0)$ converges to zero for every $x$ except on a set of linear Lebesgue measure zero.

Now it is easy to see that $\int_{-\infty}^{\infty}\left(\partial u_{n}(x, 0) / \partial x\right)^{2} d x \rightarrow 0, n \rightarrow \infty$. Hence $\mathscr{E}\left(u_{n}, u_{n}\right)$ $\rightarrow 0$ as was to be proved.

(4) Theorem 3.4 of [6].

(5) See footnote 27 of [6].

$\left.{ }^{(}\right)$The subsequent paper [9] will provide general discussions of this point. 
By means of Theorem of Appendix we get a $D$-space $(\mathscr{F}, \mathscr{E})$ on $R^{2},\left(\mathscr{F}, \mathscr{E}^{\alpha}\right)$ being the completion of $\left(\mathscr{A}, \mathscr{E}^{\alpha}\right)$ for each $\alpha>0$. This $D$-space is regular because $C_{0}^{\infty}\left(R^{2}\right) \subset \mathscr{A}$ (Corollary 1 of Appendix).

4. Equivalence of $D$-spaces. Consider a $D$-space $(X, m, \mathscr{F}, \mathscr{E})$. For $u \in L^{\infty}(X)$ $\left(=L^{\infty}(X ; m)\right)$, put $\|u\|_{\infty}=m$-ess $\sup _{x \in X}|u(x)|$. Let $L$ be a closed subalgebra of $\left(L^{\infty}(X),\|\|_{\infty}\right)$. It is well known that $L$ is then a function lattice and that $u \in L$ implies $u \wedge 1 \in L$. Therefore, by making use of Lemma 2.1(iv) and (v), we get the next lemma.

LEMMA 4.1. $\mathscr{F} \cap L$ is a function algebra and a function lattice. Further, $u \in \mathscr{F} \cap L$ implies $u \wedge 1 \in \mathscr{F} \cap L$.

Now we are in a position to define an equivalence relation in the set of all $D$-spaces.

Definition 4.1. Two $D$-spaces $(X, m, \mathscr{F}, \mathscr{E})$ and $\left(X^{\prime}, m^{\prime}, \mathscr{F}^{\prime}, \mathscr{E}^{\prime}\right)$ are called equivalent if there is an algebraic isomorphism $\Phi$ from $\mathscr{F} \cap L^{\infty}(X)$ onto $\mathscr{F}^{\prime} \cap$ $L^{\infty}\left(X^{\prime}\right)$ and $\Phi$ preserves three kinds of metrics: $\|u\|_{\infty}=\|\Phi u\|_{\infty}^{\prime}, \mathscr{E}(u, u)=\mathscr{E}^{\prime}(\Phi u, \Phi u)$ and $(u, u)_{X}=(\Phi u, \Phi u)_{X^{\prime}}$ for $u \in \mathscr{F} \cap L^{\infty}(X)$.

This definition of equivalence is the same as that of [8] where the definition is given in terms of the associated $D$-rings.

It is not difficult to see that the mapping $\Phi$ of Definition 4.1 turns out to be a lattice isomorphism and further $\Phi$ can be extended to a unitary map $\Phi_{1}$ from $(\mathscr{F}, \mathscr{E})$ onto $\left(\mathscr{F}^{\prime}, \mathscr{E}^{\prime}\right)$ and a unitary map $\Phi_{2}$ from $L_{0}^{2}(X)$ onto $L_{0}^{2}\left(X^{\prime}\right)$. Here, $L_{0}^{2}(X)$ (resp. $L_{0}^{2}\left(X^{\prime}\right)$ ) is the closure of $\mathscr{F}$ (resp. $\mathscr{F}^{\prime}$ ) in the metric space $L^{2}(X)$ (resp. $\left.L^{2}\left(X^{\prime}\right)\right)$. We can use Lemma 2.1(vi) to define the extension $\Phi_{1}$. The $L^{2}$-resolvents $\left\{G_{\alpha}, \alpha>0\right\}$ associated with equivalent $D$-spaces are mutually related by $G_{\alpha}^{\prime} u^{\prime}=$ $\Phi_{2} G_{\alpha} \Phi_{2}^{-1} u^{\prime}, u^{\prime} \in L_{0}^{2}\left(X^{\prime}\right), \alpha>0$. This relation is proved in [8].

Before proceeding to the next sections, we will summarize here some facts related to Gelfand representations of subalgebras of $L^{\infty}$. Let $(X, m)$ be as above and $L$ be a closed subalgebra of the real Banach algebra $\left(L^{\infty}(X ; m),\|\|_{\infty}\right)$. A nonzero algebraic homomorphism $\chi$ from $L$ into real numbers is called a (real) character on $L$. Denote by $\mathscr{M}$ the set of all characters on $L$. An algebraic homomorph $\Phi$ from $L$ into real functions on $\mathscr{M}$ can be defined by

$$
\Phi u(\chi)=\chi(u), \quad u \in L, \quad \chi \in \mathscr{M} .
$$

We define a neighborhood of $\chi \in \mathscr{M}$ by

$$
N\left(\chi ; u_{1}, u_{2}, \ldots, u_{n} ; \varepsilon\right)=\left\{\chi^{\prime} \in \mathscr{M} ;\left|\Phi u_{k}\left(\chi^{\prime}\right)-\Phi u_{k}(\chi)\right|<\varepsilon, k=1,2, \ldots, n\right\}
$$

with any $\varepsilon>0$ and $u_{1}, u_{2}, \ldots, u_{n} \in L$. The set $\mathscr{M}$ endowed with topology (4.2) will be called the character space of $L$.

Lemma 4.2. (i) The character space $\mathscr{M}$ of $L$ is a locally compact Hausdorff space. If the algebra $L$ is countably generated, then $\mathscr{M}$ is separable. $\mathscr{M}$ is compact if and only if $1 \in L$. 
(ii) The map $\Phi$ of (4.1) is an algebraic isomorphism and isometry from $\left(L,\|\|_{\infty}\right)$ onto $C(\mathscr{M}), C(\mathscr{M})$ being associated with the uniform norm.

(iii) Suppose that $m$ is everywhere dense $L \subset C_{b}(X)$ (the space of continuous bounded funtions on $X)$ and, for any $x \in X$, there is $a \in \in L$ with $u(x) \neq 0$. There exists then a continuous mapping $q$ from $X$ onto a dense subset of $\mathscr{M}$ characterized by

$$
\Phi u(q x)=u(x), \quad x \in X, \quad u \in L .
$$

Proof. Consider the space $A=L+(-1)^{1 / 2} L$ with uniform norm \|\|$_{\infty}$. This is a complex Banach algebra closed under the operation of taking complex conjugate function. If $u \in A$, then

$$
\frac{|u|^{2}}{1+|u|^{2}}=\frac{1}{1+a^{2}} \sum_{k=0}^{\infty}|u|^{2}\left(\frac{a^{2}-|u|^{2}}{1+a^{2}}\right)^{k} \in L
$$

where $a=\|u\|_{\infty}$. Therefore, $A$ is a symmetric algebra and the character space $\mathscr{M}$ of $L$ can be identified with the space of regular maximal ideals of $A$ (Loomis [13, subsections $23 \mathrm{~A}$ and $26 \mathrm{C}$ ]). Now statements (i) and (ii) of our lemma are known facts. The statement (iii) is evident but we give its proof here for later conveniences. Fix an $x \in X$. A map $u \rightarrow u(x)$ is clearly a character on $L$ which we denote by $q x$. $q$ is continuous at $x \in X$ because any neighborhood $N\left(\chi ; u_{1}, u_{2}, \ldots, u_{n} ; \varepsilon\right)$ of $\chi=q x$ includes the set $q(U(x))$, where $U(x)$ is an open neighborhood of $x$ defined by $U(x)=\left\{x^{\prime} \in X ;\left|u_{k}\left(x^{\prime}\right)-u_{k}(x)\right|<\varepsilon, k=1,2, \ldots, n\right\}$. Suppose that $q(X)$ is not dense in $\mathscr{M}$. There is then a nonvanishing $v \in C(\mathscr{M})$ such that $v=0$ on $q(X)$. By (ii) and (4.3), we have $\|v\|_{\infty}=\left\|\Phi^{-1} v\right\|_{\infty}=\sup _{x \in X}\left|\Phi^{-1} v(x)\right|=\sup _{x \in X}|v(q x)|=0$, which is a contradiction.

Finally we will state the following lemma according to 26J of [13].

LEMMA 4.3. Suppose that $\tilde{L}$ is a dense ideal of $L$ and every function in $\tilde{L}$ can be expressed as a difference of nonnegative functions in $\tilde{L}$. Then, for any positive linear functional $l$ on $\tilde{L}$, there exists a unique Radon measure $\mu$ on $\mathscr{M}$ such that

$$
\begin{aligned}
\Phi(\tilde{L}) & \subset L^{1}(\mathscr{M} ; \mu), \\
l(u \cdot v) & =\int_{\mathscr{M}} \Phi u(\chi) \Phi v(\chi) \mu(d \chi), \quad u \in \tilde{L}, \quad v \in L .
\end{aligned}
$$

5. Regular representations. Suppose that we are given a $D$-space $(X, m, \mathscr{F}, \mathscr{E})$. A closed subalgebra $L$ of $L^{\infty}(X ; m)$ will be said to satisfy condition (C) if it enjoys the following three properties.

(C.1) $L$ is a countably generated closed subalgebra of $L^{\infty}(X ; m)$.

(C.2) $\mathscr{F} \cap L$ is dense both in $\left(\mathscr{F}, \mathscr{E}^{\alpha_{0}}\right)$ and in $\left(L,\|\|_{\infty}\right), \alpha_{0}$ being a fixed positive number.

(C.3) $L^{1}(X ; m) \cap L$ is dense in $\left(L,\|\|_{\infty}\right)$.

THEOREM 2. (i) There exists at least one L satisfying the condition (C). (ii) Let an $L$ satisfying condition $(\mathrm{C})$ be fixed and $X^{\prime}$ be its character space. $X^{\prime}$ is compact if 
and only if $1 \in L$. There exists a regular $D$-space whose underlying space is $X^{\prime}$ and which is equivalent to the given $D$-space.

The regular $D$-space of Theorem 2(ii) will be called a regular representation of the given $D$-space with respect to the algebra $L$.

Proof of Theorem 2(i). We can find a countable subset $D_{0}$ of $C_{0}(X)$ such that each function in $C_{0}(X)$ can be uniformly approximated by a sequence of functions in $D_{0}$ whose supports are included in a suitable common compactum. $D_{0}$ is dense in $L^{2}(X ; m)$. Let $\left\{G_{\alpha}, \alpha>0\right\}$ be the $L^{2}$-resolvent associated with the given $(\mathscr{F}, \mathscr{E})$. Then, $G_{\alpha_{0}}\left(D_{0}\right) \subset \mathscr{F} \cap L^{\infty}(X ; m)$ and $G_{\alpha_{0}}\left(D_{0}\right)$ is dense in $\left(\mathscr{F}, \mathscr{E}^{\alpha_{0}}\right)$ by Lemma 2.1(i). We define $L$ as the closed subalgebra of $L^{\infty}(X ; m)$ generated by $G_{\alpha_{0}}\left(D_{0}\right)$. It is clear that this $L$ satisfies conditions (C.1) and (C.2). As for (C.3), observe that

$$
G_{\alpha_{0}}\left(D_{0}\right) \subset L^{1}(X ; m) \cap L
$$

since

$$
\begin{aligned}
\int_{X}\left|G_{\alpha_{0}} u\right| d m & \leqq \int_{X} G_{\alpha_{0}}|u| d m=\sup _{0 \leqq v \leqq 1, v \in C_{0}(X)}\left(v, G_{\alpha_{0}}|u|\right)_{X} \\
& \leqq \frac{1}{\alpha_{0}} \int_{X}|u| d m<+\infty, \quad u \in D_{0} .
\end{aligned}
$$

Proof of Theorem 2(ii). Let $L$ be a space satisfying condition (C) and $X^{\prime}$ be its character space. By (C.1) and Lemma 4.2(i), $X^{\prime}$ is a locally compact Hausdorff and separable space. $X^{\prime}$ is compact if and only if $1 \in L$. The map $\Phi$ of (4.1) is giving an algebraic isomorphism and isometry from $L$ onto $C\left(X^{\prime}\right) . \Phi$ is consequently a lattice isomorph and it holds that $\Phi(u \wedge 1)=(\Phi u) \wedge 1$ for $u \in L$. Let us put

$$
\mathscr{R}=\mathscr{F} \cap L, \quad \mathscr{R}^{\prime}=\Phi(\mathscr{R}) .
$$

Since $\mathscr{R}$ is dense in $L$ by (C.2), $\mathscr{R}^{\prime}$ is dense in $C\left(X^{\prime}\right)$. Further, by Lemma $4.1, \mathscr{R}^{\prime}$ is a lattice and $u^{\prime} \wedge 1 \in \mathscr{R}^{\prime}$ whenever $u^{\prime} \in \mathscr{R}^{\prime}$.

Keeping these in mind, we are now to construct, step by step, a regular representation $\left(X^{\prime}, m^{\prime}, \mathscr{F}^{\prime}, \mathscr{E}^{\prime}\right)$ by making use of the map $\Phi$ of (4.1).

(I) A measure $m^{\prime}$ on $X^{\prime}$. There exists a unique Radon measure $m^{\prime}$ on $X^{\prime}$ which satisfies

$$
\begin{aligned}
& \Phi\left(L^{1}(X ; m) \cap L\right) \subset L^{1}\left(X^{\prime} ; m^{\prime}\right), \\
& \int_{X} u(x) v(x) m(d x)=\int_{X^{\prime}} \Phi u\left(x^{\prime}\right) \Phi v\left(x^{\prime}\right) m^{\prime}\left(d x^{\prime}\right), \quad u \in L^{1}(X ; m) \cap L, \quad v \in L .
\end{aligned}
$$

In fact, by virtue of (C.3), we can apply Lemma 4.3 to a dense ideal $\tilde{L}=L^{1}(X ; m) \cap L$ and a positive linear functional

$$
l(u)=\int_{x} u(x) m(d x), \quad u \in \tilde{L}
$$


Consider the spaces $\mathscr{R}$ and $\mathscr{R}^{\prime}$ of (5.1). Since condition (C.2) implies that $\mathscr{R}$ is dense in $\mathscr{F}$ in $L^{2}$-sense, we have

$$
\mathscr{R} \subset L^{2}(X ; m), \quad \overline{\mathscr{R}}=L_{0}^{2}(X ; m),
$$

where the closure is taken in $L^{2}$-sense and $L_{0}^{2}(X ; m)$ denotes $\mathscr{F}$. Next we will prove

$$
\mathscr{R}^{\prime} \subset L^{2}\left(X^{\prime} ; m^{\prime}\right), \quad \overline{\mathscr{R}}^{\prime}=L^{2}\left(X^{\prime} ; m^{\prime}\right) .
$$

For any $u \in \mathscr{R}, \quad(\Phi u)^{2}=\Phi\left(u^{2}\right) \in \Phi\left(L \cap L^{1}(X ; m)\right)$ and hence $\Phi u \in L^{2}\left(X^{\prime} ; m^{\prime}\right)$ according to (5.2). In order to show that $\mathscr{R}^{\prime}$ is dense in $L^{2}\left(X^{\prime} ; m^{\prime}\right)$, take a function $u$ in $C_{0}^{+}\left(X^{\prime}\right)$. Since $\mathscr{R}^{\prime}$ is uniformly dense in $C\left(X^{\prime}\right)$ and is a lattice, we can find a $v \in \mathscr{R}^{\prime}$ and $u_{n} \in \mathscr{R}^{\prime}$ such that $0 \leqq u_{n} \leqq v$ and $u_{n}$ converges to $u$ uniformly on $X^{\prime}$. Hence, $u_{n}$ converges to $u$ in $L^{2}\left(X^{\prime} ; m^{\prime}\right)$.

Finally let us show

$$
\int_{X} u(x) v(x) m(d x)=\int_{X} u^{\prime}\left(x^{\prime}\right) v^{\prime}\left(x^{\prime}\right) m^{\prime}\left(d x^{\prime}\right), \quad u, v \in \mathscr{R},
$$

where $u^{\prime}=\Phi u$ and $v^{\prime}=\Phi v$. Take a nonnegative $v \in \mathscr{R}$. By condition (C.3) and the obvious fact that $L^{1}(X ; m) \cap L$ is a lattice, we can select $v_{n} \in L^{1}(X ; m) \cap L$ such as $0 \leqq v_{n} \leqq v m$-a.e. and $\left\|v_{n}-v\right\|_{\infty} \rightarrow 0$. Since $\Phi$ is a lattice isomorph and preserves the uniform norm, the same relations hold for $v_{n}^{\prime}$ and $v^{\prime}$. Now (5.2) for $u=v=v_{n}$ leads us to

$$
\int_{X} v(x)^{2} m(d x)=\int_{X^{\prime}} v^{\prime}\left(x^{\prime}\right)^{2} m^{\prime}\left(d x^{\prime}\right)
$$

which implies (5.5) because each element of $\mathscr{R}$ is expressed as a difference of nonnegative elements of $\mathscr{R}$ and $\Phi$ is an algebraic isomorphism.

(II) Extended map $\Phi$ on $L_{0}^{2}(X ; m)$. In view of (5.3), (5:4), and (5.5) of the preceding paragraph, the algebraic and lattice isomorphism $\Phi$ from $\mathscr{R}$ to $\mathscr{R}^{\prime}$ can be uniquely extended to

(Ф.1) A unitary map $\Phi$ from $L_{0}^{2}(X ; m)$ onto $L^{2}\left(X^{\prime} ; m^{\prime}\right)$.

Let us study the features of this extended map $\Phi$. It has the following properties.

(Ф.2) $L_{0}^{2}(X ; m)$ is a lattice and $\Phi$ is a lattice isomorphism. $\Phi(u \wedge 1)=(\Phi u) \wedge 1$ whenever $u \in L_{0}^{2}(X ; m)$.

(Ф.3) $\Phi$ is an algebraic isomorphism from $L_{0}^{2}(X ; m) \cap L^{\infty}(X ; m)$ onto

$$
L^{2}\left(X^{\prime} ; m^{\prime}\right) \cap L^{\infty}\left(X^{\prime} ; m^{\prime}\right) .
$$

Further it holds that

$$
\|u\|_{\infty}=\|\Phi u\|_{\infty}^{\prime}, \quad u \in L_{0}^{2}(X ; m) \cap L^{\infty}(X ; m) .
$$

To prove $(\Phi .2)$, take a $u \in L_{0}^{2}(X ; m)$ and find a sequence $u_{n} \in \mathscr{R}$ which converges to $u$ in $L^{2}$-sense. Since $\left|u_{n}\right| \in \mathscr{R}$ converges to $|u|$ in $L^{2}$-sense, $|u| \in L_{0}^{2}(X ; m)$. Since $\Phi$ is a lattice isomorph on $\mathscr{R}$ and preserves $L^{2}$-norm, we have $\Phi|u|=1$.i.m. $\Phi\left|u_{n}\right|$ $=1$. i.m. $\left|\Phi u_{n}\right|=|\Phi u|$. Thus we have proved the first half of (Ф.2). The latter half is similarly proved. 


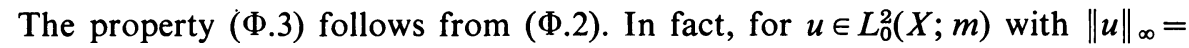
$a<+\infty$, we have $|\Phi u|=\Phi(|u|)=\Phi(|u| \wedge a)=|\Phi u| \wedge a$ which means $\|\Phi u\|_{\infty}^{\prime} \leqq\|u\|_{\infty}$. In the same way, we have $\left\|u^{\prime}\right\|_{\infty} \geqq\left\|\Phi^{-1} u^{\prime}\right\|_{\infty}$ for $u^{\prime} \in L^{2}\left(X^{\prime} ; m^{\prime}\right) \cap L^{\infty}\left(X^{\prime} ; m^{\prime}\right)$. To see that $\Phi$ is an algebraic isomorphism, take a $u \in L_{0}^{2}(X ; m) \cap L^{\infty}(X ; m)$ and a sequence $u_{n} \in \mathscr{R}$ which converges to $u$ in $L^{2}$-sense. We may assume that $\left|u_{n}\right| \leqq\|u\|_{\infty}$. Then $u_{n}^{2}$ (resp. $\left.\left(\Phi u_{n}\right)^{2}\right)$ converges to $u^{2}$ (resp. $\left.(\Phi u)^{2}\right)$ in $L^{2}$-sense. Since $\Phi$ is an algebraic isomorph on $\mathscr{R}, \Phi\left(u^{2}\right)=1 . \mathrm{i} . \mathrm{m} . \Phi\left(u_{n}^{2}\right)=(\Phi u)^{2}$.

(III) Induced D-space $\left(X^{\prime}, m^{\prime}, \mathscr{F}^{\prime}, \mathscr{E}^{\prime}\right)$. By means of the preceding map $\Phi$ on $L_{0}^{2}(X ; m) \supset \mathscr{F}$, we define

$$
\begin{aligned}
\mathscr{F}^{\prime} & =\Phi(\mathscr{F}), \\
\mathscr{E}^{\prime}\left(u^{\prime}, v^{\prime}\right) & =\mathscr{E}\left(\Phi^{-1} u^{\prime}, \Phi^{-1} v^{\prime}\right), \quad u^{\prime}, v^{\prime} \in \mathscr{F}^{\prime} .
\end{aligned}
$$

Then, $\left(X^{\prime}, m^{\prime}, \mathscr{F}^{\prime}, \mathscr{E}^{\prime}\right)$ is a $D$-space.

Condition (D.1) for $\left(X^{\prime}, m^{\prime}\right)$ has already been proved and (D.2) for $\left(\mathscr{F}^{\prime}, \mathscr{E}^{\prime}\right)$ is obvious by the property $(\Phi .1)$ of $\Phi$. Instead of proving (D.3), let us check an equivalent condition (D.3) in Remark 2.1. Take $u^{\prime} \in \mathscr{F}^{\prime}$ and put $v^{\prime}=\left(0 \vee u^{\prime}\right) \wedge 1$, $u=\Phi^{-1} u^{\prime}$. Then we have $v^{\prime}=0 \vee \Phi u \wedge 1=\Phi(0 \vee u \wedge 1)$ by (Ф.2). Since $v=$ $0 \vee u \wedge 1 \in \mathscr{F}$ and $\mathscr{E}(v, v) \leqq \mathscr{E}(u, u), v^{\prime} \in \mathscr{F}^{\prime}$ and $\mathscr{E}^{\prime}\left(v^{\prime}, v^{\prime}\right) \leqq \mathscr{E}^{\prime}\left(u^{\prime}, u^{\prime}\right)$ proving (D.3)'.

(IV) $\left(X^{\prime}, m^{\prime}, \mathscr{F}^{\prime}, \mathscr{E}^{\prime}\right)$ is equivalent to $(X, m, \mathscr{F}, \mathscr{E})$. This is evident from $(\Phi .1)$, (Ф.3) and (5.7).

(V) $\left(X^{\prime}, m^{\prime}, \mathscr{F}^{\prime}, \mathscr{E}^{\prime}\right)$ is regular. $\Phi$ preserves $\mathscr{E}^{\alpha} 0$-norm and the uniform norm on $\mathscr{R}=\mathscr{F} \cap L$. Hence by virtue of condition (C.2), $\mathscr{R}^{\prime}=\Phi(\mathscr{R})$ is dense both in $\mathscr{F}^{\prime}$ and in $C\left(X^{\prime}\right)$. Since $\mathscr{R}$ is the intersection of $\mathscr{F}$ and the uniform closure of $\mathscr{R}$, the same relation holds for $\mathscr{R}^{\prime}$ and $\mathscr{F}^{\prime}$. Therefore

$$
\mathscr{R}^{\prime}=\mathscr{F}^{\prime} \cap C\left(X^{\prime}\right) .
$$

On the other hand we have by (5.6),

$$
\sup _{x^{\prime} \in X^{\prime}}\left|u^{\prime}\left(x^{\prime}\right)\right|=m^{\prime} \text {-ess sup }\left|u^{\prime} \in X^{\prime}\left(x^{\prime}\right)\right|, \quad u^{\prime} \in \mathscr{F}^{\prime} \cap C\left(X^{\prime}\right) .
$$

Since $\mathscr{F}^{\prime} \cap C\left(X^{\prime}\right)$ is dense in $C\left(X^{\prime}\right),(5.9)$ means that $m^{\prime}$ is everywhere dense on $X^{\prime}$. The proof of $(\mathrm{V})$ is complete.

The proof of Theorem 2 has ended.

The next remarks and lemma will state the meaning of Theorem 2 for special cases.

ReMARK 5.1. Suppose that the given $D$-space $(X, m, \mathscr{F}, \mathscr{E})$ is regular. Since $m$ is everywhere dense, $C(X)$ may be considered as a closed subalgebra of $L^{\infty}(X ; m)$. Obviously $C(X)$ satisfies conditions (C.1) and (C.2). It also satisfies (C.3) because of $L^{1}(X ; m) \cap C(X) \supset C_{0}(X)$. Therefore, we may consider the regular representation with respect to $C(X)$. However, as is well known, the character space of $C(X)$ coincides with $X$ itself, and after all the regular representation goes back to the given regular $D$-space without any change. 
LEMMA 5.1. Suppose that $m$ is everywhere dense. Suppose further that an algebra $L$ satisfies not only conditions (C.1), (C.2) and (C.3) but also the following.

(C.4) $L \subset C_{b}(X)$, $L$ separates points of $X$ and, at any $x \in X$, there is $a u \in L$ such that $u(x) \neq 0$.

Let $\left(X^{\prime}, m^{\prime}, \mathscr{F}^{\prime}, \mathscr{E}^{\prime}\right)$ be the regular representation with respect to this $L$. Then,

(i) $X$ is continuously embedded onto a dense subset of $X^{\prime}$. By this embedding, any Borel set of $X$ goes to a Borel set of $X^{\prime}$ and the restriction to $X$ of any Borel set of $X^{\prime}$ is a Borel set of $X$ (with respect to the original topology).

(ii) For any Borel subset $A$ of $X^{\prime}, m^{\prime}(A)=m(A \cap X)$. Therefore, the space $\left(L^{2}\left(X^{\prime} ; m^{\prime}\right),(,)_{X^{\prime}}\right)$ is identified with the space $\left(L^{2}(X ; m),(,)_{X}\right)$.

(iii) By the above identification, $\left(\mathscr{F}^{\prime}, \mathscr{E}^{\prime}\right)$ is equal to $(\mathscr{F}, \mathscr{E})$.

Proof. By virtue of (C.4), the map $q$ of (4.3) from $X$ onto a dense subset of $X^{\prime}$ is not only continuous but also one-to-one. The rest of the lemma is obvious.

Remark 5.2. Consider the situation of Example 1 of $\S 3$. If $\partial D$ is of class $C^{\mathbf{1}}$, then the space $L=\left\{u \in C_{b}(D) ; u\right.$ is continuously extendable to $\left.\bar{D}\right\}$ satisfies conditions $($ C. 1$) \sim(C .4) .\left\{\bar{D}, d x, \mathscr{E}_{L^{2}}^{1},(,)_{D, 1}\right\}$ is just the regular representation of $\left\{D, d x, \mathscr{E}_{L^{2}}^{1},(,)_{D, 1}\right\}$ with respect to this $L$. In this case, $D$ is homeomorphically embedded into $\bar{D}$. Coming back to the general case of Lemma 5.1, $X$ is homeomorphically embedded onto a dense subset of $X^{\prime}$ if and only if for any $x_{0} \in X$ and $Y \subset X$ such as $x_{0} \notin \bar{Y}$, there exists $u \in L$ such that $u\left(x_{0}\right)=1$ and $u(x)=0$ on $Y$.

6. Strongly regular representations. Suppose that we are given a $D$-space $(X, m, \mathscr{F}, \mathscr{E})$. Denote by $\left\{G_{\alpha}, \alpha>0\right\}$ its associated $L^{2}$-resolvent.

LEMMA 6.1. (i) $G_{\alpha}$ makes the space $L^{2}(X ; m) \cap L^{\infty}(X ; m)$ invariant and

$$
\left\|G_{\alpha} u\right\|_{\infty} \leqq \frac{1}{\alpha}\|u\|_{\infty}, \quad u \in L^{2} \cap L^{\infty} .
$$

(ii) $G_{\alpha}$ makes the space $L^{\infty}(X ; m) \cap L^{1}(X ; m)\left(\subset L^{2}(X ; m)\right)$ invariant and

$$
\int_{X}\left|G_{\alpha} u(x)\right| m(d x) \leqq \frac{1}{\alpha} \int_{X}|u(x)| m(d x), \quad u \in L^{\infty} \cap L^{1} .
$$

Inequality (6.2) for $u \in C_{0}(X)$ has already been proved in the proof of Theorem 2(i). The proof for $u \in L^{\infty} \cap L^{1}$ is the same. The rest of Lemma 6.1 is clear.

Owing to Lemma 6.1(i), $G_{\alpha}$ on $L^{2} \cap L^{\infty}$ can be uniquely extended to a linear operator $\bar{G}_{\alpha}$ on $L_{0}^{\infty}(X ; m)$ (the closure of $L^{2} \cap L^{\infty}$ in $\left.L^{\infty}\right) .\left\{\bar{G}_{\alpha}, \alpha>0\right\}$ is a subMarkov resolvent on $L_{0}^{\infty}$, that is,

( $\overline{\mathrm{G}} .1)$ If $u \in L_{0}^{\infty}$ and $0 \leqq u \leqq 1 m$-a.e. then $0 \leqq \dot{\alpha} \bar{G}_{\alpha} u \leqq 1 m$-a.e.

$$
\bar{G}_{\alpha}-\bar{G}_{\beta}+(\alpha-\beta) \bar{G}_{\alpha} \bar{G}_{\beta}=0, \quad \alpha, \beta>0 .
$$

A closed subalgebra $L$ of $L_{0}^{\infty}(X ; m)$ is said to satisfy condition (R) if it enjoys the following two properties. 
(R.1) $\bar{G}_{\alpha}(L) \subset L$ for every $\alpha>0$.

(R.2) $L$ is generated by a countable subset $L_{0}$ of $\mathscr{F} \cap L$ such that each $u \in L_{0}$ is nonnegative and satisfies $\alpha \bar{G}_{\alpha+\alpha_{0}} u \leqq u, m$-a.e., $\alpha>0$.

THEOREM 3. (i) There exists an L satisfying condition (R) as well as (C). (ii) Fix an $L$ which satisfies $(\mathrm{C})$ and $(\mathrm{R})$. The regular representation of the given $D$-space with respect to this $L$ turns out to be strongly regular.

We need the next lemma for the proof of Theorem 3(i).

LEMMA 6.2. Let $S_{0}$ be a set of countable nonnegative functions in $\mathscr{F} \cap L^{\infty} \cap L^{1}$. Then, there exists a set $S$ possessing the following features.

(S.1) $S \supset S_{0}$ and $S$ is a countably generated subalgebra of $\mathscr{F} \cap L^{\infty} \cap L^{1}$. Each function of $S$ is expressed as a difference of nonnegative functions of $S$.

(S.2) For any $\alpha>0, \bar{G}_{\alpha}$ makes the space $\bar{S}$ invariant, $\bar{S}$ being the closure of $S$ in $L^{\infty}$.

Proof. According to F. Knight [11, Lemma 1], we construct $S$ as follows. Starting with $S_{0}$, assume $S_{1}, \ldots, S_{n}$ are defined. Define $S_{n+1}$ as an algebra generated by $\left\{S_{n}, G_{\alpha_{1}}\left(S_{n}\right), \ldots, G_{\alpha_{n}}\left(S_{n}\right), G_{\alpha_{n+1}}\left(S_{n}\right)\right\}$, where $\left\{a_{k}\right\}$ is the set of all positive rational numbers. Put $S=\bigcup_{n=0}^{\infty} S_{n}$, which satisfies condition (S.1) by virtue of Lemma 6.1 and of the fact that $\mathscr{F} \cap L^{\infty} \cap L^{1}$ is an algebra (Lemma 4.1). It is easy to see that condition (S.2) is met.

Proof of Theorem 3(i). Let $D_{0}^{+}$be a countable subset of $C_{0}^{+}(X)$ such that the set $D_{0}=\left\{u=u_{1}-u_{2} ; u_{i} \in D_{0}^{+}, i=1,2\right\}$ has the property in the proof of Theorem 2(i). Put $S_{0}=G_{\alpha_{0}}\left(D_{0}^{+}\right)$, which satisfies the following.

$\left(\mathrm{S}_{0} .1\right) S_{0}$ is a countable set of nonnegative functions in $\mathscr{F} \cap L^{\infty} \cap L^{1}$.

$\left(\mathrm{S}_{0} .2\right)$ The set $\left\{u=u_{1}-u_{2} ; u_{i} \in S_{0}, i=1,2\right\}$ is dense in $\left(\mathscr{F}, \mathscr{E}^{\alpha_{0}}\right)$.

$\left(\mathrm{S}_{0} .3\right) \alpha G_{\alpha+\alpha_{0}} u \leqq u m$-a.e. for $u \in S_{0}$ and $\alpha>0$.

For such an $S_{0}$, let $S$ be a set which satisfies conditions (S.1) and (S.2) of Lemma 6.2. By (S.1), there exists a set $\tilde{S}$ of countable nonnegative functions in $S$ whose linearization is just $S$. Let us put

$$
\begin{aligned}
L_{0} & =S_{0} \cup G_{\alpha_{0}}(\tilde{S}), \\
L & =\text { the closed subalgebra of } L^{\infty} \text { generated by } L_{0},
\end{aligned}
$$

then the space $L$ meets both conditions (C) and (R).

In order to check condition (C) of $\S 5$, denote by $\mathscr{R}_{0}$ the algebra generated by $L_{0}$. By $\left(\mathrm{S}_{0} .1\right),(\mathrm{S} .1)$ and Lemma $6.1, L_{0}$ and hence $\mathscr{R}_{0}$ are included in $\mathscr{F} \cap L^{\infty} \cap L^{1}$. Notice that $\mathscr{R}_{0} \subset \mathscr{F} \cap L$ and that $L$ is the closure of $\mathscr{R}_{0}$ in $L^{\infty}$. Therefore both $\mathscr{F} \cap L$ and $L^{1}(X ; m) \cap L$ are dense in $L$. Since $\mathscr{R}_{0}$ contains the set of $\left(\mathrm{S}_{0} .2\right), \mathscr{F} \cap L$ is dense in $\left(\mathscr{F}, \mathscr{E}^{\alpha_{0}}\right)$.

Coming to condition (R), it is clear that condition (R.2) is satisfied by $L_{0}$ of (6.3). Observe that $L$ is the closed subalgebra of $L^{\infty}$ generated by $S_{0} \cup \bar{G}_{\alpha_{0}}(\bar{S})$ 
By conditions (S.1) and (S.2), this means $L \subset \bar{S}$ and hence $\bar{G}_{\alpha}(L) \subset \bar{G}_{\alpha}(\bar{S})=\bar{G}_{\alpha_{0}}(\bar{S}) \subset L$ proving property (R.1) for $L$.

Proof of Theorem 3(ii). Let us fix an $L$ which satisfies conditions (C) and (R) and let $\left(X^{\prime}, m^{\prime}, \mathscr{F}^{\prime}, \mathscr{E}^{\prime}\right)$ be the regular representation with respect to $L$ according to Theorem 2(ii). We have to prove that $\left(\mathscr{F}^{\prime}, \mathscr{E}^{\prime}\right)$ is generated by a Ray resolvent kernel on $X^{\prime}$ and $\mathscr{F}^{\prime} \cap C\left(X^{\prime}\right)$ contains a set $C_{1}^{\prime}$ attached to the Ray resolvent (Definition 2.5).

A Ray resolvent can be constructed by $\Phi$ of (4.1) which is an algebraic isomorph and isometry from $L$ onto $C\left(X^{\prime}\right) . \Phi$ is a lattice isomorph and satisfies $\Phi(u \wedge 1)$ $=(\Phi u) \wedge 1$ for $u \in L$. Indeed,

$$
\begin{aligned}
\bar{G}_{\alpha}^{\prime} u^{\prime} & =\Phi \bar{G}_{\alpha} \Phi^{-1} u^{\prime}, \quad u^{\prime} \in C\left(X^{\prime}\right), \quad \alpha>0, \\
C_{1}^{\prime} & =\Phi\left(L_{0}\right)
\end{aligned}
$$

define a Ray resolvent operator $\left\{\bar{G}_{\alpha}^{\prime}, \alpha>0\right\}$ on $C\left(X^{\prime}\right)$ and a set $C_{1}^{\prime}$ attached to it.

$\bar{G}_{\alpha}^{\prime}$ is a sub-Markov resolvent on $C\left(X^{\prime}\right)$ on account of (R.1) for $L$ and $(\overline{\mathrm{G}} .1),(\overline{\mathrm{G}} .2)$ for $\bar{G}_{\alpha}$ on $L_{0}^{\infty}$. (R.2) implies that $C_{1}^{\prime}$ generates the closed algebra $C\left(X^{\prime}\right)$ and so that $C_{1}^{\prime}$ separates points of $X^{\prime}$ and, for any $x^{\prime} \in X^{\prime}$, there exists $u^{\prime} \in C_{1}^{\prime}$ nonvanishing at $x^{\prime}$. The inequalities $u^{\prime} \geqq 0, \alpha \bar{G}_{\alpha+\alpha_{0}} u^{\prime} \leqq u^{\prime}$ for $u^{\prime} \in C_{1}^{\prime}$ are obvious from (R.2).

We see that $C_{1}^{\prime}$ is included in $\mathscr{F}^{\prime} \cap C\left(X^{\prime}\right)$ because of (5.8) and (R.2).

Finally, let us prove that $\left\{\bar{G}_{\alpha}^{\prime}, \alpha>0\right\}$ generates the space $\left(\mathscr{F}^{\prime}, \mathscr{E}^{\prime}\right)$. It suffices to show

$$
\bar{G}_{\alpha}^{\prime} u=G_{\alpha}^{\prime} u, \quad m^{\prime} \text {-a.e., } \quad u \in L^{2}\left(X^{\prime} ; m^{\prime}\right) \cap C\left(X^{\prime}\right)
$$

where $\left\{G_{\alpha}^{\prime}, \alpha>0\right\}$ is the $L^{2}$-resolvent associated with $\left(\mathscr{F}^{\prime}, \mathscr{E}^{\prime}\right)$.

Observe that $G_{\alpha}^{\prime}$ is related to the $L^{2}$-resolvent $G_{\alpha}$ associated with $(\mathscr{F}, \mathscr{E})$ as follows.

$$
G_{\alpha}^{\prime} u^{\prime}=\Phi_{2} G_{\alpha} \Phi_{2}^{-1} u^{\prime}, \quad u^{\prime} \in L^{2}\left(X^{\prime} ; m^{\prime}\right)
$$

Here, $\Phi_{2}$ denotes the unitary map from $L_{0}^{2}(X ; m)$ onto $L^{2}\left(X^{\prime} ; m^{\prime}\right)$ as appeared in step (II) of the proof of Theorem 2(ii). We have indeed by (5.7), $\mathscr{E}^{\prime \alpha}\left(G_{\alpha}^{\prime} u^{\prime}, v^{\prime}\right)$ $=\left(u^{\prime}, v^{\prime}\right)_{X^{\prime}}=\left(\Phi_{2}^{-1} u^{\prime}, \Phi_{2}^{-1} v^{\prime}\right)_{X}=\mathscr{E}^{\alpha}\left(G_{\alpha} \Phi_{2}^{-1} u^{\prime}, \Phi_{2}^{-1} v^{\prime}\right)=\mathscr{E}^{\prime \alpha}\left(\Phi_{2} G_{\alpha} \Phi_{2}^{-1} u^{\prime}, v^{\prime}\right)$ for any $v^{\prime} \in \mathscr{F}^{\prime}$.

Since $\Phi$ and $\Phi_{2}$ coincide on $\mathscr{F} \cap L$ and $\bar{G}_{\alpha}$ is equal to $G_{\alpha}$ on $\mathscr{F} \cap L,(6.5)$ and (6.8) lead us to the equality (6.7) for $u^{\prime} \in \mathscr{F}^{\prime} \cap C\left(X^{\prime}\right)$. However $\mathscr{F}^{\prime} \cap C\left(X^{\prime}\right)$ is dense in $C\left(X^{\prime}\right)$. Therefore, taking sub-Markovity of $\bar{G}_{\alpha}^{\prime}$ and $G_{\alpha}^{\prime}$ into account, we get (6.7) for $u^{\prime} \in L^{2}\left(X^{\prime} ; m^{\prime}\right) \cap C\left(X^{\prime}\right)$.

The proof of Theorem 3 is complete.

The next lemma expresses the meaning of Theorem 3 for a special case.

Lemma 6.1. Suppose that $m$ is everywhere dense. Suppose further that the next condition is satisfied. 
(G.3) $(\mathscr{F}, \mathscr{E})$ is generated by a symmetric resolvent kernel $\left\{\tilde{G}_{\alpha}, \alpha>0\right\}$ on $X$ such that $\tilde{G}_{\alpha}$ transforms $C_{b}(X)$ into $C_{b}(X)$ and $\lim _{\alpha \rightarrow+\infty} \alpha G_{\alpha} u(x)=u(x)$ for any $x \in X$, $u \in C_{b}(X)$.

(i) There exists then an algebra $L$ which satisfies not only (C) and (R) but also the additional condition (C.4) of Lemma 5.1.

(ii) Let $\left(X^{\prime}, m^{\prime}, \mathscr{F}^{\prime}, \mathscr{E}^{\prime}\right)$ be the regular representation with respect to such an $L$. Then, this is strongly regular and $X$ is embedded onto a dense subset of $X^{\prime}$ in such $a$ way as Lemma 5.1. The associated Ray resolvent kernel $\bar{G}_{\alpha}^{\prime}$ on $X^{\prime}$ is an extension of $\tilde{G}_{\alpha}$ of (G.3) in the following sense. For any Borel set $A$ of $X$,

$$
\bar{G}_{\alpha}^{\prime}(x, A)=\tilde{G}_{\alpha}(x, A), \quad x \in X .
$$

Proof. (i) By replacing $L^{2}$-resolvent $\left\{G_{\alpha}\right\}$ with the smooth resolvent $\left\{\widetilde{G}_{\alpha}\right\}$ of (G.3), we can repeat the arguments of the proof of Theorem 3(i) to get an $L$ in $C_{b}(X)$. Moreover, $S_{0}(\subset L)$ separates points of $X$. In fact, assume that $\widetilde{G}_{\alpha_{0}} u(x)$ $=\widetilde{G}_{\alpha_{0}} u(y)$ for every $u \in D_{0}^{+}$. Then, it is valid for $u \in C_{b}(X)$. Hence $\alpha \widetilde{G}_{\alpha} u(x)=\alpha \widetilde{G}_{\alpha} u(y)$ for all $\alpha>0$ and $u \in C_{b}(X)$. By letting $\alpha$ tend to infinity, we have $u(x)=u(y)$, $u \in C_{b}(X)$, which means $x=y$. In the same way, we see the existence of some function of $S_{0}$ nonvanishing at any preassigned point of $X$.

(ii) The identity (6.8) is equivalent to

$$
\bar{G}_{\alpha}^{\prime} u^{\prime}(x)=\tilde{G}_{\alpha} u(x), \quad u^{\prime} \in C\left(X^{\prime}\right), \quad x \in X,
$$

where $u=\left.u^{\prime}\right|_{X}$ the restriction of $u^{\prime}$ to $X$. The right-hand side of (6.10) makes sense because $u \in C_{b}(X)$. Since (4.3) implies $\left.u^{\prime}\right|_{X}=\Phi^{-1} u^{\prime}$ for any $u^{\prime} \in C\left(X^{\prime}\right)$, we have

$$
\left.\bar{G}_{\alpha}^{\prime} u^{\prime}\right|_{X}=\Phi^{-1} \bar{G}_{\alpha}^{\prime} u^{\prime}=\Phi^{-1} \Phi \bar{G}_{\alpha} \Phi^{-1} u^{\prime}=\bar{G}_{\alpha} u, \quad u^{\prime} \in C\left(X^{\prime}\right), \quad \text { by (6.5). }
$$

However, $\bar{G}_{\alpha}$ and $\widetilde{G}_{\alpha}$ are identical on $L$ for they are on $L^{2}(X ; m) \cap C(X)$.

REMARK 6.1. We may consider that Theorem 3 treats the problem of finding strong Markov processes for a given resolvent operator. Theorem 3 solves this problem demanding that the construction procedure does not change the structure of certain associated function spaces. If we take off such a demand, we have much more possibilities of getting strong Markov processes. The proof of Theorem 3 indicates the following.

Suppose that we are given a sub-Markov resolvent operator $\left\{\bar{G}_{\alpha}, \alpha>0\right\}$ on a closed subalgebra $A$ of $B(X)$ or $L^{\infty}(X ; m)$. Here, $B(X)$ denotes the space of bounded functions with uniform norm. No kind of assumption of symmetry is imposed on $\bar{G}_{\alpha}$.

(I) If we are given a closed subalgebra $L$ of $A$ which satisfies condition (R) $\left({ }^{7}\right)$, then (6.5) defines a Ray resolvent (and consequently a strong Markov process of Ray in the sense of Remark 2.2(ii)) on the very character space $X^{\prime}$ of $L$.

$\left.{ }^{7}\right)$ Here the term of condition $(R)$ is used under a trivial modification that we do not require $L_{0}$ of (R.2) to be a subset of $\mathscr{F}$. 
(II) Let $D_{0}^{+}$be any countable subcollection of $A^{+}$. Then $D_{0}^{+}$generates an $L$ satisfying condition (R) quite in the same manner as in the proof of Theorem 3.

Our method to get $L$ which satisfies (R) is due to H. Kunita and T. Watanabe [12]. The above mentioned facts tell the generality of their method and the scope of the Ray process.

REMARK 6.2. Consider a bounded domain $D$ of $R^{N}$. The $D$-space of Example 1 of $\S 3$ meets the condition (G.3) of Lemma 6.1. According to Lemma 6.1, we get its strongly regular representation accompanied by a Ray process on an extension $D^{\prime}$ of $D$. On the other hand, we adopted in [5] the compactification $D^{*}$ of $D$ with respect to $G_{1}\left(D_{0}^{+}\right)$to serve as a state space of an extended strong Markov processa reflecting Brownian motion. This process is not necessarily a Ray's one in the strict sense of the word. However, it turns out that $\left(D^{*}, d x, \mathscr{E}_{L^{2}}^{1},(,)_{D, 1}\right)$ is a regular representation of the given $D$-space, for the algebra generated by $G_{1}\left(D_{0}^{+}\right)$ and 1 is obviously dense both in $C\left(D^{*}\right)$ and in $\mathscr{E}_{L}^{1}$.

The situation is quite the same for the $D$-space generated by each resolvent density of class $\boldsymbol{G}$ in [6].

Appendix. Construction of $D$-spaces by means of completion. Let $X$ be a locally compact Hausdorff and separable space and $m$ be a Radon measure on $X$. A pair $(\mathscr{A}, \mathscr{E})$ is said to satisfy condition $(\mathscr{A})$ if it enjoys the next three conditions.

(A.1) $\mathscr{A}$ is a linear subspace of $L^{2}(X ; m)$ and $\mathscr{E}$ is a positive definite symmetric bilinear form on $\mathscr{A}$.

(A.2) If $u \in \mathscr{A}$, then $v=(0 \vee u) \wedge 1 \in \mathscr{A}$ and $\mathscr{E}(v, v) \leqq \mathscr{E}(u, u)$.

(A.3) If $u_{n} \in \mathscr{A}$ satisfies $\left(u_{n}, u_{n}\right)_{X} \rightarrow 0$ and $\mathscr{E}\left(u_{n}-u_{m}, u_{n}-u_{m}\right) \rightarrow 0$, then

$$
\mathscr{E}\left(u_{n}, u_{n}\right) \rightarrow 0 \text {. }
$$

Condition $(\mathscr{A} .1)$ means that $\mathscr{A}$ is a real pre-Hilbert space with respect to inner product $\mathscr{E}^{\alpha}(u, v)=\mathscr{E}(u, v)+\alpha(u, v)_{X}, u, v \in \mathscr{A}$, for each $\alpha>0$.

TheOREM. Suppose that a pair $(\mathscr{A}, \mathscr{E})$ satisfies condition $(\mathscr{A})$. Let $\mathscr{F}$ be the completion of $\mathscr{A}$ with respect to a metric $\mathscr{E}^{\alpha_{0}}$ for a fixed $\alpha_{0}>0$. Then, $(X, m, \mathscr{F}, \mathscr{E})$ is a D-space.

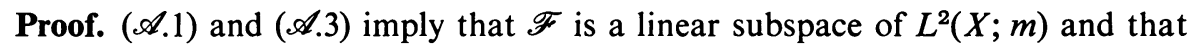
$(\mathscr{F}, \mathscr{E})$ satisfies the condition (D.2) of Definition 2.1. Therefore, for each $\alpha>0$ and $u \in L^{2}(X ; m)$, there exists $G_{\alpha} u \in \mathscr{F}$ such that $\mathscr{E}^{\alpha}\left(G_{\alpha} u, v\right)=(u, v)_{X}$ holds for any $v \in \mathscr{F}$. It suffices for us to show that $\left\{G_{\alpha}, \alpha>0\right\}$ is an $L^{2}$-resolvent, because then $(\mathscr{F}, \mathscr{E})$ coincides with the $D$-space generated by $\left\{G_{\alpha}, \alpha>0\right\}$. Obviously $\left\{G_{\alpha}, \alpha>0\right\}$ satisfies the resolvent equation. To see its sub-Markov property, let us assume that $u \in L^{2}(X ; m)$ and $0 \leqq u \leqq 1 m$-a.e.

If we put $\Phi(v)=\mathscr{E}(v, v)+\alpha(v-(1 / \alpha) u, v-(1 / \alpha) u)_{X}$ for $v \in \mathscr{F}$, then we have $\Phi(v)=\Phi\left(G_{\alpha} u\right)+\mathscr{E}^{\alpha}\left(G_{\alpha} u-v, G_{\alpha} u-v\right)$, which means that $G_{\alpha} u$ is a unique element of $\mathscr{F}$ minimizing the quadratic form $\Phi$ on $\mathscr{F}$. Further we see that $v_{n} \in \mathscr{F}$ converges 
to $G_{\alpha} u$ in $\mathscr{E}^{\alpha}$-norm if and only if $v_{n}$ is a minimizing sequence for $\Phi: \Phi\left(v_{n}\right) \rightarrow \Phi\left(G_{\alpha} u\right)$.

Since $\mathscr{A}$ is dense in $\mathscr{F}$ in $\mathscr{E}^{\alpha}$-norm, there exist $v_{n} \in \mathscr{A}$ which converges to $G_{\alpha} u$

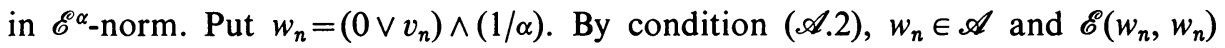
$\leqq \mathscr{E}\left(v_{n}, v_{n}\right)$. Now it is easy to see that $\Phi\left(G_{\alpha} u\right) \leqq \Phi\left(w_{n}\right) \leqq \Phi\left(v_{n}\right)$ for each $n$. However, $v_{n}$ is a minimizing sequence for $\Phi$ and so that $w_{n}$ is. Hence, $w_{n}$ converges to $G_{\alpha} u$ in $\mathscr{E}^{\alpha}$-norm and consequently a subsequence of $w_{n}$ converges to $G_{\alpha} u m$-a.e. Thus we get $0 \leqq G_{\alpha} u \leqq 1 / \alpha m$-a.e.

COROLLARY 1. In addition to the condition in Theorem, we assume that $m$ is everywhere dense on $X$ and that $\mathscr{A}$ is a dense subset of $C(X)$. Then $(X, m, \mathscr{F}, \mathscr{E})$ of the theorem is a regular D-space.

COROllary 2. Suppose that we are given a D-space $(X, m, \mathscr{F}, \mathscr{E})$. Let $\mathscr{A}$ be a subspace of $\mathscr{F}$ such that $(0 \vee u) \wedge 1 \in \mathscr{A}$ whenever $u \in \mathscr{A}$. Denote by $\mathscr{F}_{0}$ the completion of $\mathscr{A}$ with respect to $\mathscr{E}^{\alpha}{ }_{0-n o r m}$.Then, $\left(X, m, \mathscr{F}_{0}, \mathscr{E}\right)$ is a $D$-space.

COROllaRY 3. Suppose that we are given a $D$-space $(X, m, \mathscr{F}, \mathscr{E})$ with everywhere dense $m$. We assume that $\mathscr{F} \cap C_{0}(X)$ is dense in $C_{0}(X)$. Denote by $\mathscr{F}_{0}$ the com-

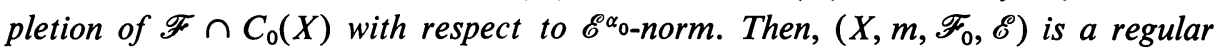
D-space.

\section{REFERENCES}

1. A. Beurling and J. Deny, Dirichlet spaces, Proc. Nat. Acad. Sci. U.S.A. 45 (1959), 208215. MR $21 \# 5098$.

2. J. Deny, Principe complet du maximum et contractions, Ann. Inst. Fourier (Grenoble) 15 (1965), fasc. 1, 259-272. MR 32 \#5913.

3. J. Deny and J. L. Lions, Les espaces du type de Beppo Levi, Ann. Inst. Fourier (Grenoble) $5(1953 / 54)$, 305-370. MR 17, 646.

4. J. L. Doob, Boundary properties of functions with finite Dirichlet integrals, Ann. Inst. Fourier (Grenoble) 12 (1962), 573-621. MR 30 \#3992.

5. M. Fukushima, A construction of reflecting barrier Brownian motions for bounded domains, Osaka J. Math. 4 (1967), 183-215. MR 37 \#6999.

6. - On boundary conditions for multi-dimensional Brownian motions with symmetric resolvent densities, J. Math. Soc. Japan 21 (1969), 58-93. MR 38 \#5291.

7. - Dirichlet spaces and their representations, Seminar on Probability 31 (1969). (Japanese)

8. —, On Dirichlet spaces and Dirichlet rings, Proc. Japan Acad. 45 (1969), 433-436.

9. - Dirichlet spaces and strong Markov processes (to appear).

10. I. M. Gel'fand, D. A. Raĭkov and G. E. Silov, Commutative normed rings, Fizmatgiz, Moscow, 1960; English transl., Chelsea, New York, 1964. MR 23 \#A1242; MR 34 \#4940.

11. F. Knight, Note on regularization of Markov processes, Illinois J. Math. 9 (1965), 548552. MR 31 \#1713.

12. H. Kunita and T. Watanabe, Some theorems concerning resolvents over locally compact spaces, Proc. Fifth Berkeley Sympos. Math. Statist. and Prob. (Berkeley, Calif., 1965/66), vol. II: Contributions to Probability Theory, part 2, Univ. of California Press, Berkeley, Calif., 1967, pp. 131-164. MR 35 \#4999. 
13. L. H. Loomis, An introduction to abstract harmonic analysis, Van Nostrand, Princeton, N. J., 1953. MR 14, 883.

14. S. Mizohata, The theory of partial differential equations, Contemporary Math., no. 9, Iwanami Shoten, Tokyo, 1965. (Japanese) MR 38 \#396.

15. D. Ray, Resolvents, transition functions, and strongly Markovian processes, Ann. of Math. (2) 70 (1959), 43-72. MR 21 \#6027.

16. T. Shiga and T. Watanabe, On Markov chains similar to the reflecting barrier Brownian motion, Osaka J. Math. 5 (1968), 1-33. MR 39 \#7676.

\section{TOKYo UNIVERSITY OF EdUCATION,}

TOKYO, JAPAN

UNIVERSITY OF ILLINOIS,

URBANA, ILLINOIS 61801 